\title{
Immediate visualization of recombination events and chromosome segregation defects in fission yeast meiosis
}

\author{
Dmitriy $\mathrm{Li}^{1,2} \cdot$ Marianne Roca $^{1,3} \cdot$ Raif Yuecel $^{1,2} \cdot$ Alexander Lorenz $^{1}$ (D)
}

Received: 1 November 2018 /Revised: 8 January 2019 / Accepted: 10 January 2019/Published online: 9 February 2019

(C) The Author(s) 2019

\begin{abstract}
Schizosaccharomyces pombe, also known as fission yeast, is an established model for studying chromosome biological processes. Over the years, research employing fission yeast has made important contributions to our knowledge about chromosome segregation during meiosis, as well as meiotic recombination and its regulation. Quantification of meiotic recombination frequency is not a straightforward undertaking, either requiring viable progeny for a genetic plating assay, or relying on laborious Southern blot analysis of recombination intermediates. Neither of these methods lends itself to high-throughput screens to identify novel meiotic factors. Here, we establish visual assays novel to $S z$. pombe for characterizing chromosome segregation and meiotic recombination phenotypes. Genes expressing red, yellow, and/or cyan fluorophores from spore-autonomous promoters have been integrated into the fission yeast genomes, either close to the centromere of chromosome 1 to monitor chromosome segregation, or on the arm of chromosome 3 to form a genetic interval at which recombination frequency can be determined. The visual recombination assay allows straightforward and immediate assessment of the genetic outcome of a single meiosis by epi-fluorescence microscopy without requiring tetrad dissection. We also demonstrate that the recombination frequency analysis can be automatized by utilizing imaging flow cytometry to enable high-throughput screens. These assays have several advantages over traditional methods for analyzing meiotic phenotypes.
\end{abstract}

Keywords Schizosaccharomyces pombe - Chromosome segregation · Meiotic recombination · Spore-autonomous promoters · Imaging flow cytometry

\section{Introduction}

Meiosis is a highly conserved process that produces haploid sex cells (gametes) as an integral part of sexual reproduction

This article is part of a Special Issue on Recent advances in meiosis from DNA replication to chromosome segregation "edited by Valérie Borde and Francesca Cole, co-edited by Paula Cohen and Scott Keeney".

Electronic supplementary material The online version of this article (https://doi.org/10.1007/s00412-019-00691-y) contains supplementary material, which is available to authorized users.

Alexander Lorenz

a.lorenz@abdn.ac.uk

1 Institute of Medical Sciences (IMS), University of Aberdeen, Foresterhill, Aberdeen AB25 2ZD, UK

2 Iain Fraser Cytometry Centre (IFCC), University of Aberdeen, Foresterhill, Aberdeen AB25 2ZD, UK

3 Present address: Laboratoire de Biologie du Développement de Villefranche-sur-Mer (LBDV), Sorbonne Université, 06230 Villefranche-sur-Mer, France
(Hunter 2015). During meiosis, chromosomes are deliberately broken to initiate homologous (meiotic) recombination that physically connects the equivalent maternal and paternal (homologous) chromosomes; this is absolutely essential for correct chromosome segregation (Petronczki et al. 2003; Lam and Keeney 2015). Only if these connections (chiasmata) are achieved accurately, healthy gametes containing a single chromosome complement will result from the two meiotic cell divisions. In the process, homologous chromosomes are reshuffled and genes are re-assorted; this provides the genetic diversity that makes individuals unique. Failure to perform meiosis correctly has been shown to cause infertility, miscarriages, and hereditary disorders in mammals (Hassold and Hunt 2001); meiosis is thus fundamental to sexual reproduction.

Meiotic recombination is initiated by Spo11, a topoisomerase VI-like transesterase, creating meiotic double-stranded DNA breaks (DSBs) (Lam and Keeney 2015). These DSBs are subsequently repaired by homology-directed repair mechanisms driven by the RecA-family recombinases Rad51 and Dmc1. Rad51 and its meiosis-specific paralogue Dmc1 are 
supported by a host of ancillary factors through loading Rad51 and/or Dmc1 onto a processed DSB site and stabilizing them as multimeric nucleoprotein filaments. These ancillary factors include Rad51 paralogues (Gasior et al. 1998; Grishchuk and Kohli 2003; Bleuyard et al. 2005; Sasanuma et al. 2013; Brown and Bishop 2014; Lorenz et al. 2014; Abreu et al. 2018), and factors evolutionarily unrelated to RecA, such as Rad52, Swi5-Sfr1, and Hop2-Mnd1 (Gasior et al. 1998; Chen et al. 2004; Ellermeier et al. 2004; Zierhut et al. 2004; Petukhova et al. 2005; Kerzendorfer et al. 2006; Vignard et al. 2007; Octobre et al. 2008). In Sz. Pombe, the Hop2Mnd1 orthologues are called Meu13-Mcp7, and similar to the situation in other eukaryotes, meiotic recombination is strongly reduced in their absence (Nabeshima et al. 2001; Saito et al. 2004). Homology-directed repair can follow several pathways, and ultimately results in crossover (CO) and non-crossover recombination outcomes (Phadnis et al. 2011; Hunter 2015). Only COs between homologous chromosomes support the formation of chiasmata, which together with sister chromatid cohesion are needed for proper chromosome segregation (Marston 2014). Cohesion is achieved by the cohesin complex which physically entraps the sister chromatids right after their replication during S phase (Nasmyth and Haering 2009). Cohesin holds sister chromatids together until all chromosomes are properly attached to microtubules in metaphase, at which point the kleisin subunit of cohesin is destroyed and anaphase ensues (Nasmyth and Haering 2009; Marston 2014). To reduce the diploid chromosome complement to a haploid one, meiosis consists of two cell divisions following a single round of DNA replication; special modifications to sister chromatid cohesion have to be in place to enable this. During meiosis I, homologous chromosomes are segregated from each other, and cohesins are only removed from the chromosome arms, whereas cohesins at centromeres remain protected for the second meiotic division. During meiosis II, centromeric cohesin protection is removed to allow sister chromatids to be segregated from each other (Petronczki et al. 2003; Marston 2014). A key centromeric protector is the Mei-S332 homolog Shugoshin, Sgo1 (Katis et al. 2004; Kitajima et al. 2004; Marston et al. 2004; Rabitsch et al. 2004), and the absence of Sgol and chiasmata, indeed, generates a strong chromosome segregation defect during meiosis (Hirose et al. 2011).

Here, we establish and characterize visual assays to quantify chromosome segregation defects and meiotic recombination frequency which are new to $S z$. pombe. Visual assays for determining meiotic recombination frequencies were originally established in Arabidopsis, and more recently adapted for budding yeast (Francis et al. 2007; Thacker et al. 2011). These visual recombination assays utilize genes encoding red, yellow, and cyan fluorophores driven by gamete-specific promoters, and are integrated at specific loci on a given chromosome to form genetic intervals. The four products (gametes) of a single meiosis will fluoresce in a color corresponding to the fluorophore gene(s) they receive. In Arabidopsis, the fluorophores are expressed from the pollen-specific post-meiotic LAT52 promoter, and various genetic intervals (fluorescent-tagged lines, FTLs) have been generated and adopted widely (e.g., Yelina et al. 2013; Séguéla-Arnaud et al. 2017; Kurzbauer et al. 2018). Also, the budding yeast version of the visual recombination assay starts to enjoy popularity and several recent studies used spore-autonomous fluorophore expression to determine meiotic recombination frequency (e.g., Vincenten et al. 2015; Arter et al. 2018; González-Arranz et al. 2018; Raffoux et al. 2018; Rogers et al. 2018). In yeasts, this kind of setup allows assessment of the frequency of exchange of flanking markers (COs) and has advantages over traditional methods for studying meiotic recombination - such as using nutritional markers (White and Petes 1994; Smith 2009) or Southern blotting of DNA from meiotic yeast cells (Hyppa and Smith 2009; Oh et al. 2009): (I) spores can be assessed regardless of their viability (ability to form a visible yeast colony), (II) the simplicity of this method will allow its use for high-throughput genetic screens, and (III) achieving large sample sizes is straightforward when using imaging flow cytometry. Additionally, this can also be used as a tool for monitoring chromosome segregation defects, when different fluorophore markers are inserted close to a centromere (Thacker et al. 2011; this study).

These visual assays represent a novel, powerful, and easyto-use experimental tool for fission yeast allowing straightforward analysis of chromosome segregation and homologous recombination defects during meiosis. They also enable the identification and characterization of complex phenotypes (single and double $\mathrm{CO}$ formation) in high-throughput screens via imaging flow cytometry.

\section{Materials and methods}

\section{Molecular and microbiological techniques}

Plasmids and details of construction are given in Table S1. DNA-modifying enzymes (high-fidelity DNA polymerase Q5, Taq DNA polymerase, T4 DNA ligase, restriction endonucleases) and the NEBuilder HiFi DNA Assembly Master Mix were obtained from New England BioLabs (NEB), Inc. (Ipswich, MA, USA), and the In-fusion HD Cloning kit from Takara Bio, Inc. (Mountain View, CA, USA). Oligonucleotides (Table S2) were supplied by SigmaAldrich Co. (St. Louis, MO, USA). All relevant regions of plasmids were verified by DNA sequencing (Source BioScience plc, Nottingham, UK). Plasmid sequences are available as supporting online material (Lorenz 2018).

Escherichia coli was grown in LB and SOC media, when appropriate media contained $100 \mu \mathrm{g} / \mathrm{ml}$ ampicillin (Sambrook and Russell 2000). Competent E. coli XL1-blue cells (Agilent 
Technologies, Santa Clara, CA, USA) were transformed following the protocol provided by the manufacturer.

Schizosaccharomyces pombe strains (Table S3) were cultured on yeast extract (YE), and on yeast nitrogen base glutamate (YNG) agar plates containing the required supplements (concentration $250 \mu \mathrm{g} / \mathrm{ml}$ on YE, and $75 \mu \mathrm{g} / \mathrm{ml}$ on YNG). Crosses were performed on malt extract (ME) agar with the required amino acids (concentration $50 \mu \mathrm{g} / \mathrm{ml}$ ). Fission yeast transformations were performed using a standard Li-acetate protocol (Brown and Lorenz 2016). Construction of the hphMX4-marked meu13 $\Delta-22$ strain UoA585 by marker swap from meu13 $\Delta:$ ura $^{+}$has been described elsewhere (Lorenz 2015); the meu13 $\Delta-43::$ natMX4 strain UoA723 was derived by transforming an appropriate marker swap cassette amplified by PCR (oligonucleotides oUA101 and oUA102, Table S2) from pALo121 into UoA585 (meu13A22::hphMX4) (Lorenz 2015; Brown and Lorenz 2016). Strains carrying the meu13 $\Delta-22$, meu13 $\Delta-43, \operatorname{sgol} \Delta$, and rec12 $\Delta-169$ alleles were derived by crossing from UoA585, UoA723, JG17888, and GP3717, respectively (Davis and Smith 2003; Gregan et al. 2005; Lorenz 2015). A natMX6marked partial deletion of ade6 (ade6-3' $\triangle:$ :natMX6) was created by cloning natMX6 from pCR2.1-nat as an EcoRI-fragment between the EcoRI site within the coding sequence and the EcoRI site downstream of the STOP codon of ade6 on plasmid pALo159 (Table S1). The cassette was released from the resulting plasmid (pALo169) by a HindIII-EcoRV restriction digest (Table S1), and transformed into strain ALP729 (Table S3). This generated strain UoA570 (Table S3) carrying a natMX6-marked 848 bp deletion at ade6, removing 429 bp of coding sequence. All ade6-3' $\triangle$ ::natMX6 strains have been derived from UoA570 by crossing. Spore-autonomously expressed fluorophore genes were targeted to their intended sites using flanking homologous DNA sequences which were provided via various strategies (Bähler et al. 1998; Matsuyama et al. 2004; Gregan et al. 2006) (Tables S1 and S3).

All sequence details and positional information about $S z$. pombe genomic loci have been extracted from https://www. pombase.org (Wood et al. 2002).

Spore viability by random spore analysis and meiotic recombination assays have been performed as previously described (Osman et al. 2003; Smith 2009; Sabatinos and Forsburg 2010; Lorenz et al. 2012).

\section{Microscopy}

For microscopy cells from sporulating cultures were suspended in sterile demineralized water, and spotted onto microscopic slides. After placing a cover slip over the cell suspension, cells were immobilized by squashing the slide in a filter paper block, and afterwards the cover slip was sealed with clear nail varnish. Microscopic analysis was done using a
Zeiss Axio Imager.M2 (Carl Zeiss AG, Oberkochen, Germany) epi-fluorescence microscope equipped with the appropriate filter sets to detect red, yellow, and cyan fluorescence. A $63 \times$ objective (Plan-Apochromat, aperture 1.4) was used for taking black-and-white images with a Zeiss AxioCam MRm CCD camera controlled by AxioVision 40 software v4.8.2.0. For chromosome segregation experiments 9-20 and for recombination assays 20-25 randomly selected fields of view were photographed and evaluated. Images were pseudo-colored and overlaid using Adobe Photoshop CC (Adobe Systems Inc., San José, CA, USA). Images of mature four-spored asci were evaluated manually; data was collected and analyzed in Microsoft Excel 2016 MSO (version 16.0.4738.1000, 32-bit).

\section{Imaging flow cytometry}

The ImageStreamX Mark II (Merck KGaA, Darmstadt, Germany) is an imaging flow cytometer, where an image of each individual cell is acquired as it flows through the cytometer. It measures hundreds of thousands of individual cells in minutes, combining the high-throughput capabilities of conventional flow cytometry with single-cell imaging. The ImageStream measures not only total fluorescence intensities but also the spatial image of the fluorescence plus bright-field and dark-field images of each cell in a population.

For a more extensive overview of data acquisition and analysis in ImageStreamX, see Basiji (2016). Briefly, the INSPIRE acquisition software generates raw image data (.rif file) without compensation which can then be directly loaded into IDEAS for further analysis. Using the IDEAS software, the .rif files will then be converted into compensated image files (.cif) by applying the compensation matrix (.ctm) generated from single fluorescence controls during the acquisition. The file resulting from analysis is stored as .daf (data analysis file), which is used for plotting features derived from the bright-field, dark-field, and fluorescence single cell images in the form of histograms or bivariate scatter plots. Subpopulations are generated using these plots and saved as analysis template for further datasets.

For imaging flow cytometry, cellular material containing asci was suspended in 1× PBS, pH $7.5(8 \mathrm{~g} / 1 \mathrm{NaCl}, 2 \mathrm{~g} / 1 \mathrm{KCl}$, $1.15 \mathrm{~g} / 1 \mathrm{Na}_{2} \mathrm{HPO}_{4} \cdot 7 \mathrm{H}_{2} \mathrm{O}, 2 \mathrm{~g} / 1$ anhydrous $\left.\mathrm{KH}_{2} \mathrm{PO}_{4}\right)$, harvested by centrifugation $(6000 \times \mathrm{g}, 30 \mathrm{~s})$, and re-suspended in $1 \times$ PBS, pH 7.5. Data was acquired on the ImageStreamX Mark II using INSPIRE acquisition software (Merck kGaA). Cellular parameters were measured in Channel 1 (Brightfield, BF), Channel 2 (GFP*, a yellow-shifted version of green fluorescent protein, using a $485 \mathrm{~nm}$ laser), Channel 4 (RFP, red fluorescent protein, $561 \mathrm{~nm}$ ), Channel 7 (CFP, cyan fluorescent protein, $405 \mathrm{~nm}$ ), and Channel 12 (side scatter, $785 \mathrm{~nm}$ ) with magnification set to $60 \times$. Briefly, objects of interest (asci) with a BF "area" of 50 to $200 \mu^{2}$ and an "aspect ratio" (ratio 
of minor axis to major axis) lower than 0.5 ("doublet area") were selected. Focused cells were identified by a "gradient RMS" feature value of 50 or higher. A typical file contained about 25,000 focused yeast cells.

Data evaluation for identification of asci and spore phenotyping were performed using IDEAS software (version 6.2; Merck). A focused population of asci were identified within the "doublet area" and based on the features "Modulation" for fluorescent channels (the Modulation texture feature measures the intensity range of an image, normalized between 0 and 1) and "Intensity" for side scatter (SSC) using the custom masks "Morphology" and "Object(right)," respectively. Further refinement was performed each on RFP, GFP*, and CFP fluorescence via "Intensity." Following analysis of the merged triple fluorescent population using "Length" and "Elongatedness" (ratio of the height over width of the object's bounding mask) features (custom BF mask "AdaptiveErode, M01, Ch01, 75") resulted in identification of asci of interest. Finally, spore phenotype analysis was conducted by evaluating the fluorescent area using custom masks for each fluorescent intensity (GFP* intensity 200-4095, RFP intensity 754095, and CFP intensity 150-4095) and by applying Boolean algebra to identify particular combinations of fluorescent colors. Asci with a mask area larger than $3 \mu \mathrm{m}^{2}$ were considered positive for a particular spore phenotype.

\section{Results and discussion}

\section{Identifying spore-autonomous promoters in Schizosaccharomyces pombe}

To test whether a particular upstream regulatory sequence is a spore-autonomous promoter (Thacker et al. 2011), we cloned a 700-931-bp region upstream of the start codon of the $S z$. pombe eis 1, pil2, eng2, agn2, and mde10 genes in front of a cyan (mCerulean) or red (tdTomato) fluorophore gene inserted in pDUAL, a vector restoring leu ${ }^{+}$by integrating at the leu1-32 mutant locus (Matsuyama et al. 2004). Fluorophore genes were terminated by Saccharomyces spp. $P G K 1$ downstream regulatory sequence: $T_{P G K 1}$ from $S$. bayanus for mCerulean, and $T_{P G K 1}$ from $S$. kudriavzevii for tdTomato (Thacker et al. 2011). The candidate promoters were selected on the basis of its corresponding gene being upregulated during late meiosis or sporulation (Mata et al. 2002): eng2, agn2, and mde10 code for proteins involved in spore wall formation, eis 1 encodes an eisosome assembly protein, and pil2 a component of the eisosome. The promoter of $S$. cerevisiae YKL050c has previously been shown to support spore-autonomous expression of fluorophores in budding yeast (Thacker et al. 2011); Sz. pombe eis 1 is the single homolog of the $S$. cerevisiae paralogue pair EIS1 and YKL050c. The resulting plasmids (pALo139, pALo140, pALo141,
pALo142, pALo175; Table S1) were digested with ApaI to release the $l e u 1^{+}$integration cassettes containing the constructs; these were transformed into $h^{+}$and $h^{-}$fission yeast strains (ALP729 and FO652) carrying the leu1-32 mutation. Two leu $1^{+}$strains of different mating types carrying differently colored fluorophore constructs were crossed to each other, and presence or absence of spore-specific fluorescence was recorded on an epi-fluorescence microscope. $P_{\text {eng2 } 2}, P_{\text {agn } 2}$, and $P_{m d e 10}$ failed to produce fluorescence levels visible under the microscope (data not shown). $P_{\text {eis } 1}$ and $P_{\text {pil } 2}$ were strong spore-autonomous promoters yielding clear red or cyan fluorescence in spores of mature asci (data not shown).

To avoid ectopic recombination events between the $P_{\text {eis } 1}$ and $P_{\text {pil2 }}$ constructs and the upstream regions of endogenous eis 1 and pil2, we decided to follow a similar strategy as Keeney and co-workers (Thacker et al. 2011), and investigated whether $P_{e i s 1}$ and $P_{\text {pil }}$ from Schizosaccharomyces species other than $S z$. pombe can be used as spore-autonomous promoters in $S z$. pombe. Indeed, the upstream sequences of the Sz. japonicus eis1 and pil2 homologs SJAG_04227 and $S J A G \_02707$, as well as the regions upstream of $S z$. cryophilus and Sz. octosporus pil2 homologs SPOG_00147 and SOCG_04642, cloned in front of fluorophores produced strong fluorescence in spores of $S z$. pombe asci (Fig. 1a). $P_{\text {SJAG_04227 }}, P_{\text {SPOG_00147, }}$ and $P_{\text {SOCG_04642 }}$ were selected to drive tdTomato (red fluorescence protein, from now called RFP), GFP* (yellow-shifted green fluorescence protein, terminated by $T_{P G K 1}$ from $S$. mikatae) (Griesbeck et al. 2001; Thacker et al. 2011), and mCerulean (cyan fluorescence protein, from now on called CFP) expression in all experimental constructs (Fig. 1).

\section{Monitoring meiosis chromosome segregation defects}

Markers inserted next to the centromere can be used to monitor meiotic chromosome segregation defects. Previously, this has been exploited in genetic screens by introducing bacterial operator repeats ( $\mathrm{lacO}$ or tetO) close to centromeres in budding and fission yeast, to identify chromosome segregation mutants via the distribution of LacI- or TetR-GFP fusions binding to their respective operators, thus becoming visible as small foci (Straight et al. 1996; Michaelis et al. 1997; Nabeshima et al. 1998; Katis et al. 2004; Rabitsch et al. 2004; Gregan et al. 2005). Introducing spore-autonomously expressed fluorophore markers with different colors at the centromere (Figs. 1b and 2a) has the advantages of (I) enabling distinction of meiosis I and meiosis II segregation defects in a single assay (Fig. 2) rather than requiring homozygous and heterozygous setups of $l a c O$ or tet $O$ repeats integrated close to a centromere, and (II) likely not interfering with chromosome behavior as strongly as lacO or tet $O$ repeats (Fuchs et al. 2002; Sofueva et al. 2011). Fission yeast asci are ordered; due to the physical constraints of the zygotic cell 
Fig. 1 Spore-autonomous expression of fluorophores. a Schematic and examples of main constructs, $P_{\text {SOCG } 04642}-G F P^{*}$ $T_{P G K 1 \text { (mik) }}$ from strain UoA694, $P_{S P O G \_00147-m C e r u l e a n-}$

$T_{P G K 1 \text { (bay) }}$ from strain UoA727,

$P_{S P O G \text { 00147 }}$-tdTomato- $T_{P G K 1(k u d)}$ from strain UoA726, and $P_{S J A G_{-}}$ 04227-tdTomato- $T_{P G K 1 \text { (kud) }}$ from strain UoA694; scale bar in example images represents $10 \mu \mathrm{m}$. b Plasmid maps of CEN1targeting (CEN1t) constructs using the Sz. octosporus $S P O G$ 00147 (pil2) promoter to drive RFP (tdTomato) and CFP (mCerulean) expression. c Plasmid maps of constructs usable for generating genetic intervals (see main text for details); RFP is driven by the $S z$.japonicus SJAG_04227 (eis 1) promoter in pALo148 and by $S z$. octosporus SPOG 00147 (pil2) promoter in pALo181, CFP by the $S z$. octosporus SPOG_00147 (pil2) promoter in pALo1 68 \& pALo182, and the yellow-shifted GFP* by the $S z$. cryophilus SOCG 04642 (pil2) promoter in pALo179 \& pALo186 a

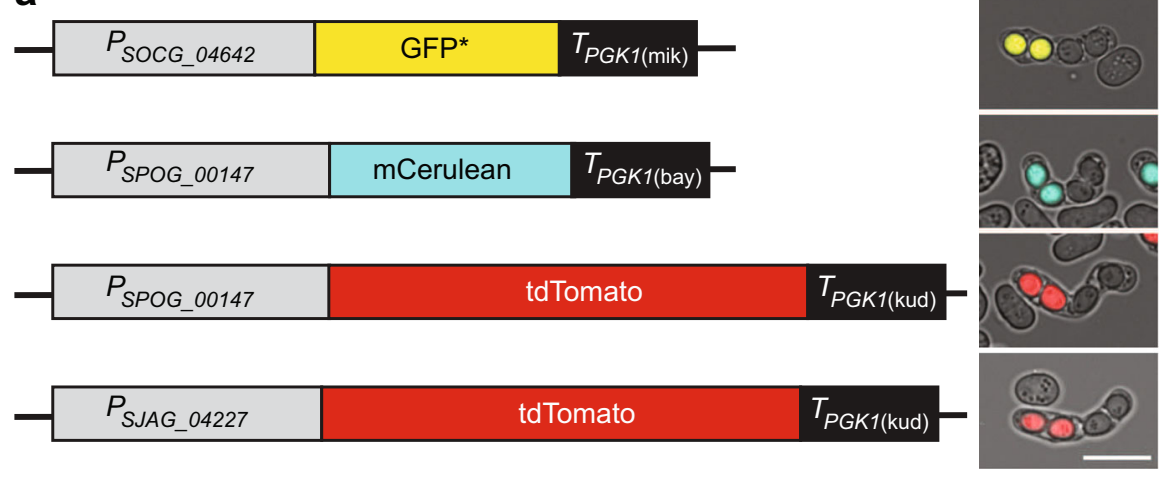

b
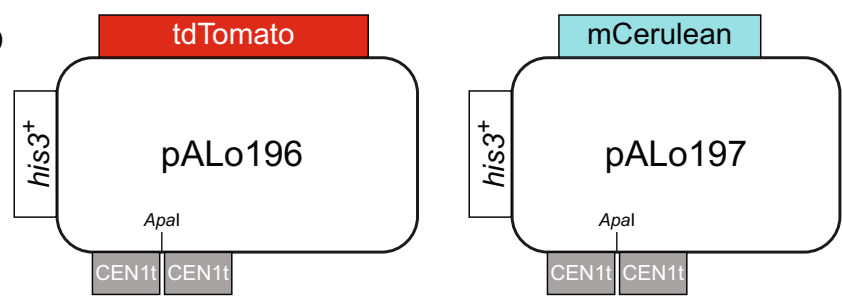

C
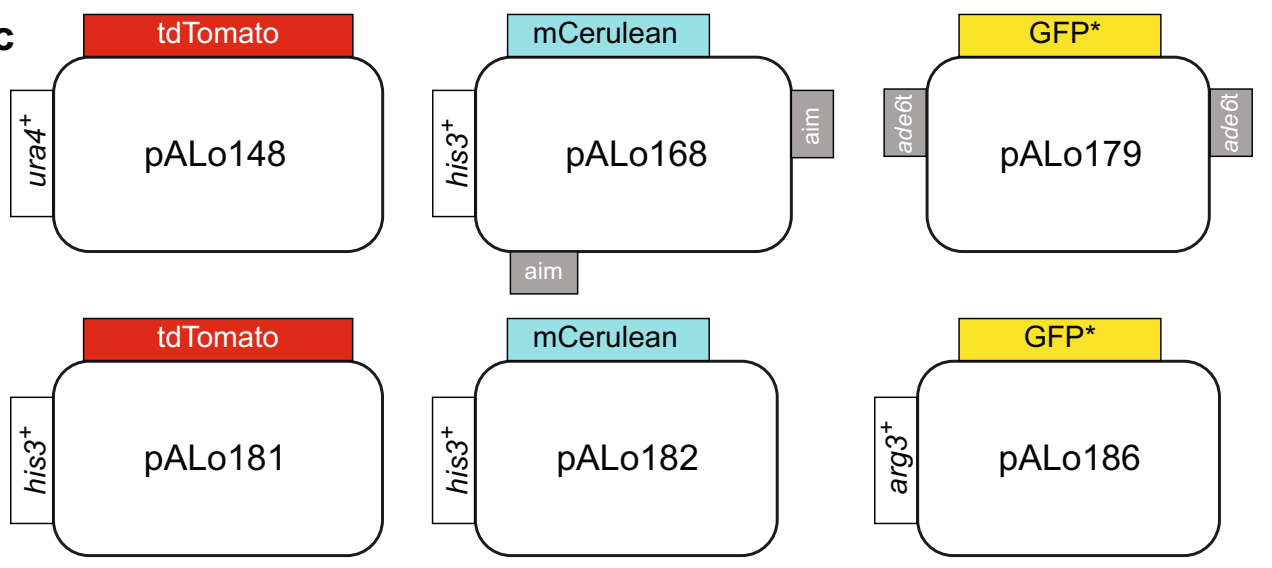

size and shape, microtubular spindles can orientate only along the longitudinal axis of the zygote, which means that the neighboring nuclei/spores in one half of the zygote are the sister products generated in meiosis II (Fig. 2b). This makes the evaluation of chromosome mis-segregation a comparatively straightforward undertaking in $S z$. pombe.

The integration of spore-autonomously expressed fluorophore cassettes at the centromere of chromsome 1 (CEN1) was enabled by sequences homologous to a genomic region downstream of the perl (SPAP7G5.06) locus (position $3,751,911$ on chromosome 1), similar to a strategy developed for high-throughput gene deletion in fission yeast (Gregan et al. 2006). The CEN1-targeting plasmids carrying a his $3^{+}$ selection marker and the spore-autonomously expressed fluorophore $P_{S P O G \text { 00147-tdTomato (pALo196) or }}$

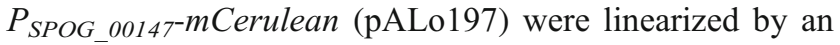
ApaI restriction digest and transformed into yeast strains ALP729 or FO652 (Tables S1 and S3). All strains carrying CEN1::P SPOG_00147-tdTomato were generated by crossing from UoA726 (ALP729 transformed with ApaI-digested
pALo196), and all strains carrying CEN1::P SPOG_00147mCerulean were derived from UoA727 (FO652 transformed with ApaI-digested pALo197) by crossing.

We tested the functionality of our assay carrying fluorophore genes under the control of the sporeautonomous SPOG_00147-promoter integrated close to CEN1 (Fig. 2a) with a set of mutants defective in meiotic recombination (meu13, spol1) and/or kinetochore function (sgol) (Keeney et al. 1997; Nabeshima et al. 2001; Sharif et al. 2002; Rabitsch et al. 2004). For this, we only evaluated four-spored asci, and ignored asci with spore counts of 1, 2, or 3 , to exclude incidences of clear nuclear division failures in meiosis I or II. As expected, in wild-type and meu13A crosses, chromosome 1 is correctly segregated, in almost all cases (Fig. 2c). We did observe a low frequency (3.3\%) of $\mathrm{CO}$ recombination between the fluorophore marker and the physical centromere in wild type, leading to red-cyan pairs of sister nuclei, rather than red-red and cyan-cyan pairs (Fig. 2c). In meu13 $\Delta$, which strongly reduces meiotic recombination (Nabeshima et al. 2001), no COs were observed, but two 


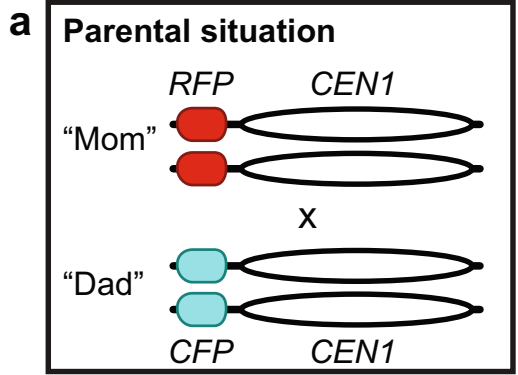

b

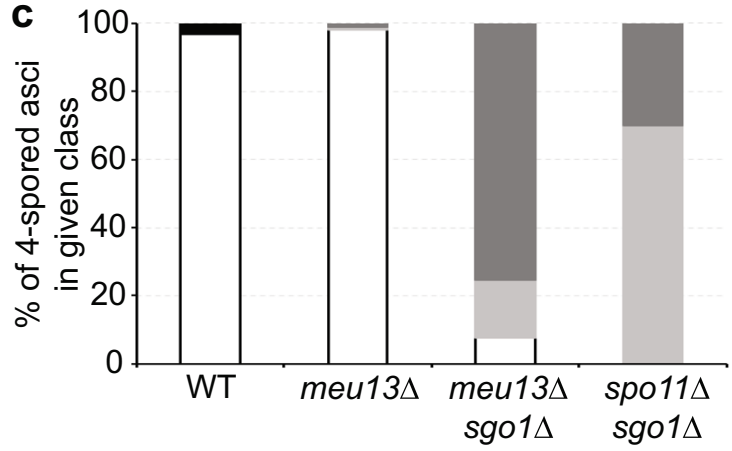

Fig. 2 Chromosome segregation assay using spore-autonomous expression of fluorophores. a Schematic of assay, RFP and CFP are expressed from the Sz. octosporus SPOG_00147 (pil2) promoter integrated at position $3,751,911$ on chromosome 1 downstream of the per 1 (SPAP7G5.06) locus close to its centromere (CEN1). b Meiotic nuclear divisions generate an ordered tetrad with sister nuclei from meiosis II (MII) ending up next to one another. c Chromosome segregation phenotypes in fourspored wild-type (WT; UoA726 $\times$ UoA727, $n=274$ ), meu $13 \Delta$ $(\mathrm{UoA} 752 \times \mathrm{UoA755}, n=101)$, meu13 $\Delta$ sgol $\Delta(\mathrm{UoA} 756 \times \mathrm{UoA759}$, $n=53)$, and spoll $\Delta$ sgol $\Delta(\mathrm{UoA} 760 \times \mathrm{UoA} 763, n=20$ asci) asci. A low frequency of crossover (CO) events $(3.3 \%)$ between the fluorophore genes and CEN1 has been observed in WT incidences of chromosome mis-segregation could be recorded (Fig. 2c). As an obvious example for meiotic chromosome mis-segregation, we employed double mutants of sgol $\Delta$ with meu $13 \Delta$ or spoll $\Delta$. A sgol $\Delta$ single mutant does not produce a strong mis-segregation phenotype (Rabitsch et al. 2004), but in combination with the absence of recombination factors, a meiotic non-disjunction phenotype can be observed (Hirose et al. 2011). Indeed, massive chromosome segregation defects are obvious in asci of meul3 $\Delta$ sgol $\Delta$ and spoll $\Delta$ sgol $\Delta$ double mutants (Fig. 2c). In spoll $\Delta$ sgol $\Delta$, the percentage meiotic non-disjunction is slightly higher than in meul3 $\Delta$ $\operatorname{sgol} \Delta$, and there are also more meiosis I chromosome missegregation events in spoll $\Delta$ sgol $\Delta$. In meul3 $\Delta$, chromosome segregation can presumably be supported to some degree, because a small number of chiasmata is still being produced, whereas in spoll $\Delta$ meiotic DSB formation is completely abrogated and thus no chiasmata are formed.

\section{Creating a genetic interval with fluorophore markers to assess meiotic recombination frequency}

To explore whether fluorophore markers inserted at defined genomic sites on a single chromosome to create a genetic interval that can be used to determine meiotic recombination frequencies, we transformed constructs integrating on chromosome 3 forming a genetic interval of $\sim 45 \mathrm{~kb}$ around the ade6 locus (Fig. 3a) (Osman et al. 2003; Lorenz et al. 2012). To target the $\mathrm{ura}^{+}{ }^{+}$-marked $P_{\text {SJAG_0422 }}$-tdTomato construct to the same locus as $u r a 4^{+}$-aim 2 on chromosome 3 (at position $1,291,583, \sim 26.5 \mathrm{~kb}$ upstream of ade6), a $u r a 4^{+}-P_{\text {SJAG_04227- }}$ tdTomato- $T_{P G K 1 \text { (Skud) }}$ cassette was amplified by PCR from pALo148 adding $\sim 80 \mathrm{bp}$ of homologous flanking sequences (oligonucleotides oUA113 and oUA114, Table S2) (Bähler et al. 1998), and transformed into strain FO652. All strains

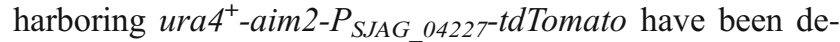
rived from the resulting transformant, UoA523 (Table S3), by crossing. A similar approach failed to deliver the his $3^{+}$-

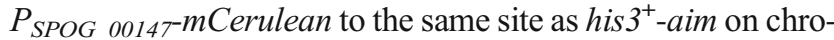
mosome 3 (at position 1,337,447, $19.5 \mathrm{~kb}$ downstream of ade6). Therefore, we cloned larger homologous flanking sequence up- and downstream of his $3^{+}-P_{S P O G} 0014^{-}$ $m$ Cerulean- $T_{P G K 1 \text { (Sbay) }}$ into the Not I sites of pALo182 (Table S1). The backbone and insert (his $3^{+}-P_{S P O G} 00147^{-}$ $m$ Cerulean- $\left.T_{P G K 1(\text { Sbay) }}\right)$ of pALo182 after a Not I digest were merged with a 436-bp upstream (oligonucleotides oUA189 and oUA190) and a 646-bp downstream flanking sequence (oligonucleotides oUA191 and oUA192, Table S2) amplified by PCR from $S z$. pombe genomic DNA (strain MCW1196, Table S3) in a single NEBuilder assembly reaction (in the process, the NotI sites flanking the whole construct were replaced by SmaI sites). The whole cassette was excised from the resulting plasmid (pALo168, Table S1) by SmaI digestion 
a

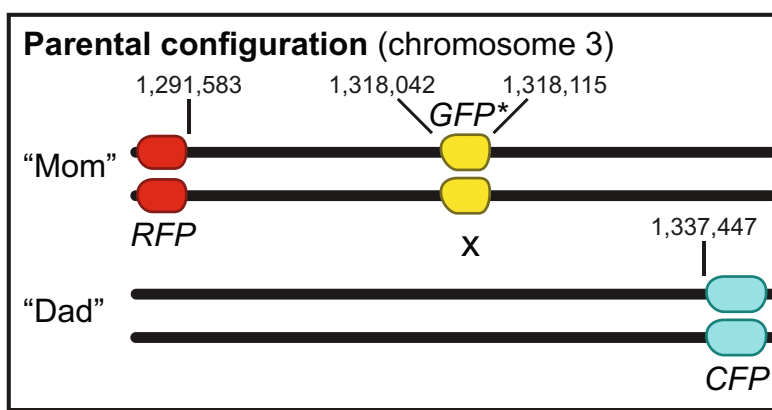

b

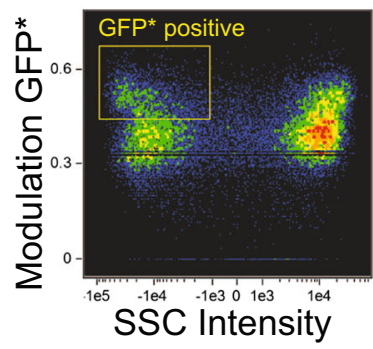

C

Parental configuration (NOT CFP AND RFP AND YFP)

\section{CO between $G F P^{*}$ and CFP (YFP AND RFP AND CFP)}

\section{CO between RFP and GFP* (NOT YFP AND RFP AND CFP)}

\section{double CO (2-chromatid) (NOT RFP AND YFP AND CFP)}

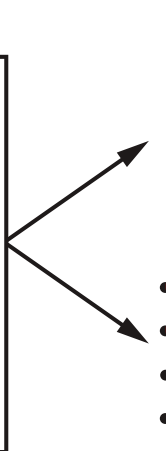

Triple fluorescent

Positive selected

Based on RFP, GFP* \& CFP intensity

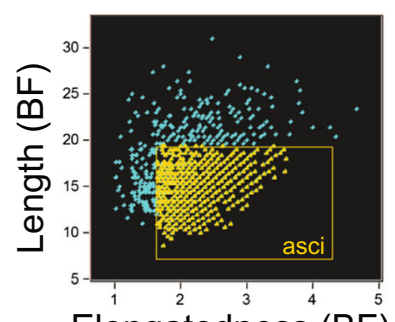

Elongatedness (BF)

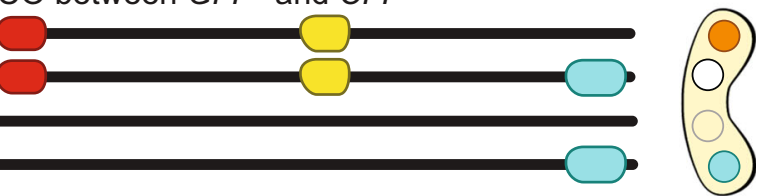

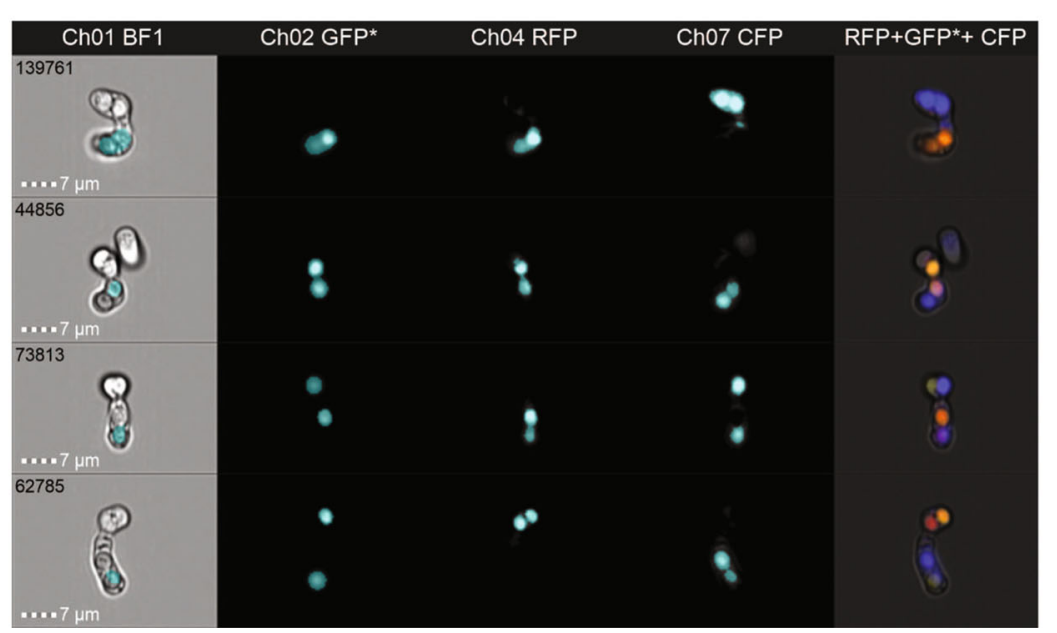

Fig. 3 Genetic interval constructed with spore-autonomously expressed fluorescent markers can be analyzed by imaging flow cytometry. a Schematic of the genetic interval constructed: RFP expressed from the Sz. japonicus SJAG_04227 (eisl) promoter together with a ra $^{+}{ }^{+}$marker is integrated on chromosome 3 at position 1,291,583, the same site as ura $4^{+}$-aim 2 (Osman et al. 2003); CFP expressed from the $S z$. octosporus SPOG_00147 (pil2) promoter together with a his $3^{+}$marker is integrated on chromosome 3 at position 1,337,447, the same site as his $3^{+}$-aim (Osman et al. 2003); GFP* expression driven from the Sz. cryophilus SOCG_04642 (pil2) promoter, the construct is integrated between positions 1,318,042 and 1,318,115 on chromosome 3 (downstream of ade6 at its endogenous locus). Only outcomes of single crossovers (COs)

between the three markers are shown; double COs are rare (see Figs. S2 and $\mathrm{S} 3$ for double COs observed in this kind of assay). Please note that order of spore colors is not fixed, but can rotate perpendicular to the meiotic spindle axis. b Outline of the workflow to identify asci based on particular cellular features on the Amnis ImageStreamX Mark II. Modulation measures the intensity range of an image normalized between 0 and 1 by calculating Modulation $=($ Max Pixel - Min Pixel $) /($ Max Pixel + Min Pixel). c Examples of ascus phenotypes from a cross of wild-type strains (UoA694 $\times$ UoA676) as shown in a; Boolean algebra mask equations used to discriminate between the different ascus types as presented in $\mathrm{Ch} 01 \mathrm{BF} 1$

and transformed into strain ALP729. This generated strain UoA676 (Table S3), from which all strains carrying his $3^{+}$aim- $P_{S P O G} 00147^{-m}$ Cerulean were derived by crossing. Finally, the yellow spore marker ( $P_{S O C G} 04642-G F P^{*}$ $\left.T_{P G K 1 \text { (Smik) }}\right)$ on pALo179 was generated by an NEBuilder assembly of $P_{S O C G} 04642$ (PCR on genomic DNA of $S z$. octosporus yFS286, oligonucleotides oUA201 and oUA202) and $G F P^{*}-T_{P G K 1(S m i k)}$ (PCR on pSK726, oligonucleotides oUA204 and oUA138) between the ade6-targeting sequences on pALo159 (linearized by a BamHI and BglII digest) (Tables S1 and S2). The ade $6^{+}:: P_{S O C G} 04642^{-G F P *}$ strain UoA666 (Table S3) was created by transforming the ade6targeting cassette from pALo179 (amplified by PCR, oligonucleotides oUA142 and oUA143; Table S2) into the 
ade6-3' $\triangle:$ :natMX6 strain UoA570. This transformation restored ade $6-3^{\prime} \Delta$ to ade $\sigma^{+}$and removed the natMX6 cassette,

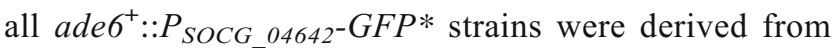
UoA666 by crossing.

As this assay visualizes recombination events, we evaluated it using standard epi-fluorescence microscopy, and also tested whether single-cell imaging flow cytometry (Basiji 2016) could be exploited to perform high-throughput screens with the spore-autonomously expressed fluorophore recombination assay. We established a workflow on the Amnis ImageStreamX Mark II imaging flow cytometer to select for mature asci displaying fluorescence from a mixed population of cells in a standard cross (mature fluorescing asci, immature non-fluorescing asci, zygotes, vegetative cells), and subsequently applied customizations in the software to identify spore color phenotypes unique for the recombination outcomes we expected to occur in this assay (Fig. 3b, c).

The important first steps are identifying focused cells by using a measure of the "gradient RMS" feature of the brightfield image to define the focus quality. Single and multiple cells were determined by plotting the cell mask area versus cell mask aspect ratio, whereby the asci were located in the doublet area. Once focused subspecies are identified via gating, they were used as the starting point to analyze recombination products by determining the spore phenotype, which is only feasible by utilizing the fluorescent markers GFP*, RFP, and CFP.

For this purpose, mainly the "Modulation (texture)" feature was applied to objectively discriminate the bright fluorescence pattern of GFP*, RFP, and CFP associated asci. We first gated GFP*-positive objects on the basis of the appropriate "Modulation (texture)" against darkfield using the side scatter (SSC) parameter in a bivariate plot. In the next stage, the gated GFP* population was subanalyzed for the modulation of RFPand CFP-containing spores (Fig. 3b).

Employing the ability of the IDEAS software for creating Boolean logic, masks with good determination of spore borders in each fluorescent channel were selected, and these advanced combined masks determined spores with particular fluorescent phenotypes (Fig. 3c). For example, spores with all three fluorescent proteins are only possible, if recombination happened between GFP* and CFP, whereas spores containing RFP and CFP are only possible, if recombination happened between RFP and GFP*. Finally, asci were quantified within the triple merged combined fluorescent populations by using the newly created shape features "Length" versus "Elongatedness" in brightfield. Thereby, asci with recombination products were identified within a "Length" $<20$ and an "Elongatedness" > 2 (Fig. 3).

If a particular experimental setup requires a distinction between four-spored asci and asci with irregular spore numbers $(1,2,3,>4)$, masks using information from the brightfield channel can be programmed to accommodate this.
Because the fluorophore markers were inserted at the same positions as the nutritional markers of an established recombination assay (Figs. 4a, b and S1a, b) (Osman et al. 2003; Lorenz et al. 2012, 2014), we could directly compare the outcomes of the different assays assessed by various methods. We used two slightly different recombination assays utilizing nutritional markers: one contained a point mutation at ade6 (ade6-704, a T645A substitution mutation; Park et al. 2007), the other one a dominant drug resistance marker inserted at the $3^{\prime}$ end of ade6 creating a partial deletion (ade6-3'A::natMX6). We used the latter to test whether a drastically different recombination frequency is caused by introducing a heterologous piece of DNA into the genetic interval. The natMX6 cassette is $\sim 1.25 \mathrm{~kb}$ in size and removes 848 bp of genomic DNA at ade6 (429 bp of which are ade6 coding sequence); in comparison, the spore-autonomously expressed GFP $^{*}$ cassette is $\sim 2.1 \mathrm{~kb}$ in size and inserted just downstream of the ade6 open reading frame (removing $73 \mathrm{bp}$ just downstream of the 3 '-untranslated region of ade6).

Despite all these differences between the markers, the recombination frequencies within the genetic intervals were remarkably similar (Figs. 4c and S1c). The genetic intervals with the nutritional markers produced $11.88 \%$ (ade6-704) and $13.33 \%$ (ade6-3' $\Delta$ ) COs, respectively (Figs. 4c, Table S4). The interval with the fluorophore markers measured $9.41 \%$ COs on the epi-fluorescence microscope and $14.57 \%$ COs on the imaging flow cytometer (Fig. 4c, Table S5). The results were comparable, when the ade6- or $G F P^{*}$-markers were initially linked with his $3^{+}$-aim or CFP, respectively $(10.63 \% \mathrm{CO}$ for ade6-704, 8.33\% CO for ade6-3' $\Delta, 7.68 \% \mathrm{CO}$ for fluorophore markers evaluated by epi-fluorescence microscopy; Fig. S1, Tables S4 and S5). In all types of assays, we could also detect a few rare double $\mathrm{CO}$ events (Figs. 4 and S1, Tables S4 and S5). Because asci can be evaluated as an ordered tetrad in the fluorophore-based assay (Figs. 2b and 3a), information about the involvement of two, three, or all four chromatids in the double $\mathrm{CO}$ can be extracted. Within the four double $\mathrm{CO}$ events over the two slightly different genetic intervals evaluated on the epi-fluorescence microscope (Figs. 4b and S1b), examples for participation of two, three, or four chromatids could be found (Figs. S2 and S3). The observed frequency of double $\mathrm{CO}$ in any of the genetic assays is equal with or slightly higher than expected from the frequency in neighboring intervals (Tables S4 and S5), in line with Sz. pombe not displaying CO interference (Munz 1994).

In a meu 13 mutant meiotic intra- and intergenic recombination is strongly decreased (Nabeshima et al. 2001). When running the fluorophore-based assay in a meu13 $\Delta$ background, as expected, a 3.6- to 5.7-fold reduction in $\mathrm{CO}$ formation could be observed (Fig. 4c). No double COs were detected in the meu $13 \Delta$ crosses. This demonstrates that in $S z$. pombe a genetic interval consisting of spore-autonomously expressed fluorescent markers behaves very similarly to a genetic interval built from nutritional markers. 


\section{a Parental configuration - Plating}

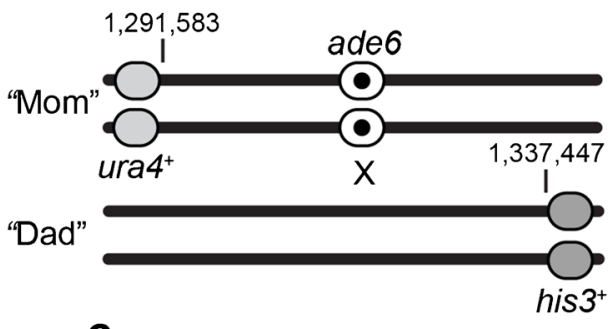

C

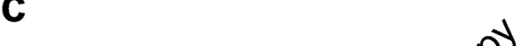

b

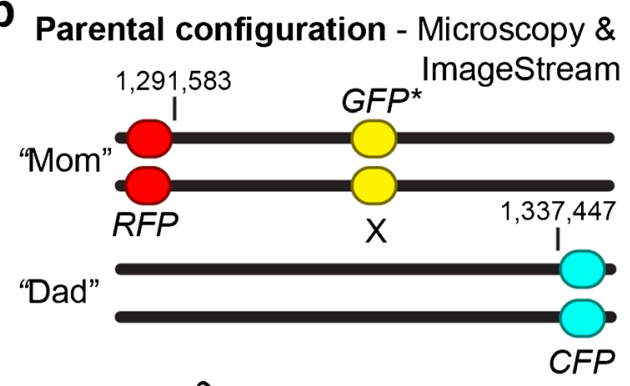

Fig. 4 Comparison of genetic intervals generated by nutritional markers and spore-autonomously expressed fluorescent markers. a Schematic of genetic recombination assay using nutritional markers and plating of colonies. In UoA112, the ade6 marker is a point mutation (ade6-704) without hotspot activity; in UoA736, it is a partial deletion of ade6 by integrating a natMX6 cassette (ade6-3' $\Delta$ ). In both instances, ade6 is at its endogenous locus on chromosome 3 , and the position for the coding sequence is $1,316,337-1,317,995$. The flanking markers $u r a 4^{+}$and his $3^{+}$are the artificially introduced markers (aim) and his $3^{+}$-aim, which have been previously described (Osman et al. 2003); ura $4^{+}$-aim2 is integrated on chromosome 3 at position 1,291,583, and his $3^{+}$-aim at position $1,337,447$. b Schematic of spore-autonomously expressed fluorophore recombination assay (see also Fig. 3a), the RFP gene is at the same

\section{Conclusion}

Here, we established assays employing spore-autonomously expressed fluorescent proteins to determine meiotic chromosome mis-segregation and meiotic recombination frequencies in the fission yeast, $S z$. pombe. We generated a series of plasmids containing selectable markers in addition to the sporespecific fluorophores (Fig. 1, Table S1); this makes the whole

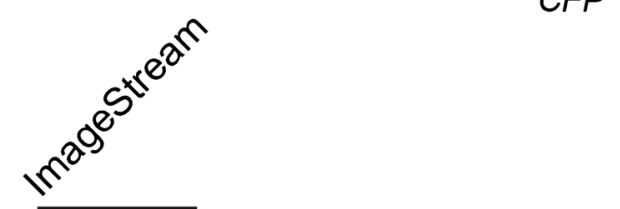

double $\mathrm{CO}$

single $\mathrm{CO}$ position as $u r a 4^{+}$-aim 2 in a, the $C F P$ gene at the same position as his $3^{+}$-aim in a, and the GFP* gene is inserted downstream of ade $6^{+}$. c Results from recombination assays in a and $\mathbf{b}$ : crossover $(\mathrm{CO})$ recombinant frequencies were determined in wild-type (WT) crosses by random spore analysis for the plating assay (a), using data from $n=3$ independent crosses with 160 progeny each. CO recombinant frequencies were determined in WT and meu13 $\Delta$ crosses either by counting manually on an epifluorescence microscope (UoA694 $\times$ UoA676 $n=356$ asci, UoA742 $\times$ UoA743 $n=305$ asci) or by high-throughput single cell assessment on an imaging flow cytometer (ImageStream) (UoA694 $\times$ UoA676 $n=916$ asci, UoA742 $\times$ UoA743 $n=370$ asci). Please note that ImageStream can only identify one out of two double CO classes

system portable enabling the creation of genetic intervals at virtually any position within the $S z$. pombe genome.

Ectopic spore-autonomous promoters from $S z$. japonicus work in $S z$. pombe; this raises the possibility that expression from this type of regulatory elements is conserved, and could be used to develop a similar system in Sz.japonicus. This is of interest, because $S z$. japonicus produces 8-spored asci (an additional mitosis following the two meiotic divisions) (Klar 
2013) enabling an even better resolution of genetic events. We validated our system by comparison to an established recombination assay (Osman et al. 2003; Lorenz et al. 2012, 2014) utilizing nutritional markers (Fig. 4), and demonstrated that imaging flow cytometry can be used to run genetic highthroughput screens for recombination phenotypes (Figs. 3 and 4). Due to its portability and advantages over existing assays, our fluorophore-based system represents a novel addition to the ever-growing genetic toolkit for probing the cell biology of fission yeast.

Acknowledgements We are grateful to Scott Keeney, Franz Klein, Jürg Kohli, Josef Loidl, Kim Nasmyth, Ken E. Sawin, Gerald R. Smith, Takashi Toda, Matthew C. Whitby, and the National BioResource Project (NBRP) Japan for providing strains and/or plasmids; to M.N. Asogwa, A. Bebes, and L. Duncan for technical assistance; and to M. De Carvalho for spotting a critical typographical error in an earlier version of the manuscript. Microscopy was performed at the University of Aberdeen Microscopy \& Histology facility (Kevin Mackenzie). This work was supported by a Carnegie Trust for the Universities of Scotland Research Incentive Grant (No. 70021), and the University of Aberdeen (College of Life Sciences and Medicine Start-up grant).

Open Access This article is distributed under the terms of the Creative Commons Attribution 4.0 International License (http:// creativecommons.org/licenses/by/4.0/), which permits unrestricted use, distribution, and reproduction in any medium, provided you give appropriate credit to the original author(s) and the source, provide a link to the Creative Commons license, and indicate if changes were made.

Publisher's note Springer Nature remains neutral with regard to jurisdictional claims in published maps and institutional affiliations.

\section{References}

Abreu CM, Prakash R, Romanienko PJ, Roig I, Keeney S, Jasin M (2018) Shu complex SWS1-SWSAP1 promotes early steps in mouse meiotic recombination. Nat Commun 9:3961. https://doi.org/10.1038/ s41467-018-06384-x

Arter M, Hurtado-Nieves V, Oke A, Zhuge T, Wettstein R, Fung JC, Blanco MG, Matos J (2018) Regulated crossing-over requires inactivation of Yen1/GEN1 resolvase during meiotic prophase I. Dev Cell 45:785-800.e6. https://doi.org/10.1016/j.devcel.2018.05.020

Bähler J, Wu JQ, Longtine MS, Shah NG, Mckenzie III A, Steever AB, Wach A, Philippsen P, Pringle JR (1998) Heterologous modules for efficient and versatile PCR-based gene targeting in Schizosaccharomyces pombe. Yeast 14:943-951. https://doi.org/ 10.1002/(SICI)1097-0061(199807)14:10<943::AID-YEA292>3.0. $\mathrm{CO} ; 2-\mathrm{Y}$

Basiji DA (2016) Principles of Amnis imaging flow cytometry. Methods Mol Biol 1389:13-21. https://doi.org/10.1007/978-1-4939-3302-0_2

Bleuyard J-Y, Gallego ME, Savigny F, White CI (2005) Differing requirements for the Arabidopsis Rad51 paralogs in meiosis and DNA repair. Plant J 41:533-545. https://doi.org/10.1111/j.1365-313X. 2004.02318.x

Brown MS, Bishop DK (2014) DNA strand exchange and RecA homologs. Cold Spring Harb Perspect Biol 7:a016659. https://doi.org/10. 1101/cshperspect.a016659

Brown SD, Lorenz A (2016) Single-step marker switching in Schizosaccharomyces pombe using a lithium acetate transformation protocol. Bioanalysis 6:e2075. https://doi.org/10.21769/BioProtoc. 2075

Chen Y-K, Leng C-H, Olivares H, Lee MH, Chang YC, Kung WM, Ti SC, Lo YH, Wang AHJ, Chang CS, Bishop DK, Hsueh YP, Wang TF (2004) Heterodimeric complexes of Hop2 and Mnd1 function with Dmc1 to promote meiotic homolog juxtaposition and strand assimilation. Proc Natl Acad Sci U S A 101:10572-10577. https:// doi.org/10.1073/pnas.0404195101

Davis L, Smith GR (2003) Nonrandom homolog segregation at meiosis I in Schizosaccharomyces pombe mutants lacking recombination. Genetics 163:857-874

Ellermeier C, Schmidt H, Smith GR (2004) Swi5 acts in meiotic DNA joint molecule formation in Schizosaccharomyces pombe. Genetics 168:1891-1898. https://doi.org/10.1534/genetics.104.034280

Francis KE, Lam SY, Harrison BD, Bey AL, Berchowitz LE, Copenhaver GP (2007) Pollen tetrad-based visual assay for meiotic recombination in Arabidopsis. Proc Natl Acad Sci U S A 104:3913-3918. https://doi.org/10.1073/pnas.0608936104

Fuchs J, Lorenz A, Loidl J (2002) Chromosome associations in budding yeast caused by integrated tandemly repeated transgenes. J Cell Sci 115:1213-1220

Gasior SL, Wong AK, Kora Y, Shinohara A, Bishop DK (1998) Rad52 associates with RPA and functions with Rad55 and Rad57 to assemble meiotic recombination complexes. Genes Dev 12:2208-2221

González-Arranz S, Cavero S, Morillo-Huesca M, Andújar E, PérezAlegre M, Prado F, San-Segundo P (2018) Functional impact of the H2A.Z histone variant during meiosis in Saccharomyces cerevisiae. Genetics 209:997-1015. https://doi.org/10.1534/ genetics.118.301110

Gregan J, Rabitsch PK, Sakem B, Csutak O, Latypov V, Lehmann E, Kohli J, Nasmyth K (2005) Novel genes required for meiotic chromosome segregation are identified by a high-throughput knockout screen in fission yeast. Curr Biol 15:1663-1669. https://doi.org/10. 1016/j.cub.2005.07.059

Gregan J, Rabitsch PK, Rumpf C, Novatchkova M, Schleiffer A, Nasmyth K (2006) High-throughput knockout screen in fission yeast. Nat Protoc 1:2457-2464. https://doi.org/10.1038/nprot. 2006.385

Griesbeck O, Baird GS, Campbell RE, Zacharias DA, Tsien RY (2001) Reducing the environmental sensitivity of yellow fluorescent protein. Mechanism and applications. J Biol Chem 276:29188-29194. https://doi.org/10.1074/jbc.M102815200

Grishchuk AL, Kohli J (2003) Five RecA-like proteins of Schizosaccharomyces pombe are involved in meiotic recombination. Genetics 165:1031-1043

Hassold T, Hunt P (2001) To err (meiotically) is human: the genesis of human aneuploidy. Nat Rev Genet 2:280-291. https://doi.org/10. 1038/35066065

Hirose Y, Suzuki R, Ohba T, Hinohara Y, Matsuhara H, Yoshida M, Itabashi Y, Murakami H, Yamamoto A (2011) Chiasmata promote monopolar attachment of sister chromatids and their co-segregation toward the proper pole during meiosis I. PLoS Genet 7:e1001329. https://doi.org/10.1371/journal.pgen.1001329

Hunter N (2015) Meiotic recombination: the essence of heredity. Cold Spring Harb Perspect Biol 7:a016618. https://doi.org/10.1101/ cshperspect.a016618

Hyppa RW, Smith GR (2009) Using Schizosaccharomyces pombe meiosis to analyze DNA recombination intermediates. Methods Mol Biol 557:235-252. https://doi.org/10.1007/978-1-59745-527-5_15

Katis VL, Galova M, Rabitsch KP, Gregan J, Nasmyth K (2004) Maintenance of cohesin at centromeres after meiosis I in budding yeast requires a kinetochore-associated protein related to MEI-S332. Curr Biol 14:560-572. https://doi.org/10.1016/j.cub.2004.03.001

Keeney S, Giroux CN, Kleckner N (1997) Meiosis-specific DNA doublestrand breaks are catalyzed by Spo11, a member of a widely conserved protein family. Cell 88:375-384 
Kerzendorfer C, Vignard J, Pedrosa-Harand A, Siwiec T, Akimcheva S, Jolivet S, Sablowski R, Armstrong S, Schweizer D, Mercier R, Schlögelhofer P (2006) The Arabidopsis thaliana MND1 homologue plays a key role in meiotic homologous pairing, synapsis and recombination. J Cell Sci 119:2486-2496. https://doi.org/10. $1242 /$ jcs. 02967

Kitajima TS, Kawashima SA, Watanabe Y (2004) The conserved kinetochore protein shugoshin protects centromeric cohesion during meiosis. Nature 427:510-517. https://doi.org/10.1038/nature02312

Klar AJS (2013) Schizosaccharomyces japonicus yeast poised to become a favorite experimental organism for eukaryotic research. G3 3: 1869-1873. https://doi.org/10.1534/g3.113.007187

Kurzbauer M-T, Pradillo M, Kerzendorfer C, Sims J, Ladurner R, Oliver C, Janisiw MP, Mosiolek M, Schweizer D, Copenhaver GP, Schlögelhofer P (2018) Arabidopsis thaliana FANCD2 promotes meiotic crossover formation. Plant Cell 30:415-428. https://doi. org $/ 10.1105 /$ tpc. 17.00745

Lam I, Keeney S (2015) Mechanism and regulation of meiotic recombination initiation. Cold Spring Harb Perspect Biol 7:a016634. https:// doi.org/10.1101/cshperspect.a016634

Lorenz A (2015) New cassettes for single-step drug resistance and prototrophic marker switching in fission yeast. Yeast 32:703-710. https://doi.org/10.1002/yea.3097

Lorenz A (2018) Plasmid sequences - immediate visualization of recombination events and chromosome segregation defects in fission yeast meiosis. figshare. doi: https://doi.org/10.6084/m9.figshare.7264673

Lorenz A, Osman F, Sun W, Nandi S, Steinacher R, Whitby MC (2012) The fission yeast FANCM ortholog directs non-crossover recombination during meiosis. Science 336:1585-1588. https://doi.org/10. 1126/science. 1220111

Lorenz A, Mehats A, Osman F, Whitby MC (2014) Rad51/Dmc1 paralogs and mediators oppose DNA helicases to limit hybrid DNA formation and promote crossovers during meiotic recombination. Nucleic Acids Res 42:13723-13735. https://doi.org/10.1093/ nar/gku1219

Marston AL (2014) Chromosome segregation in budding yeast: sister chromatid cohesion and related mechanisms. Genetics 196:31-63. https://doi.org/10.1534/genetics.112.145144

Marston AL, Tham W-H, Shah H, Amon A (2004) A genome-wide screen identifies genes required for centromeric cohesion. Science 303(80):1367-1370. https://doi.org/10.1126/science.1094220

Mata J, Lyne R, Burns G, Bähler J (2002) The transcriptional program of meiosis and sporulation in fission yeast. Nat Genet 32:143-147. https://doi.org/10.1038/ng951

Matsuyama A, Shirai A, Yashiroda Y, Kamata A, Horinouchi S, Yoshida M (2004) pDUAL, a multipurpose, multicopy vector capable of chromosomal integration in fission yeast. Yeast 21:1289-1305. https://doi.org/10.1002/yea.1181

Michaelis C, Ciosk R, Nasmyth K (1997) Cohesins: chromosomal proteins that prevent premature separation of sister chromatids. Cell 91: 35-45

Munz P (1994) An analysis of interference in the fission yeast Schizosaccharomyces pombe. Genetics 137:701-707

Nabeshima K, Nakagawa T, Straight AF, Murray A, Chikashige Y, Yamashita YM, Hiraoka Y, Yanagida M (1998) Dynamics of centromeres during metaphase-anaphase transition in fission yeast: Dis1 is implicated in force balance in metaphase bipolar spindle. Mol Biol Cell 9:3211-3225

Nabeshima K, Kakihara Y, Hiraoka Y, Nojima H (2001) A novel meiosisspecific protein of fission yeast, Meu13p, promotes homologous pairing independently of homologous recombination. EMBO J 20: 3871-3881. https://doi.org/10.1093/emboj/20.14.3871

Nasmyth K, Haering CH (2009) Cohesin: its roles and mechanisms. Annu Rev Genet 43:525-558. https://doi.org/10.1146/annurevgenet-102108-134233
Octobre G, Lorenz A, Loidl J, Kohli J (2008) The Rad52 homologs Rad22 and Rtil of Schizosaccharomyces pombe are not essential for meiotic interhomolog recombination, but are required for meiotic intrachromosomal recombination and mating-type-related DNA repair. Genetics 178:2399-2412. https://doi.org/10.1534/genetics. 107.085696

Oh SD, Jessop L, Lao JP, Allers T, Lichten M, Hunter N (2009) Stabilization and electrophoretic analysis of meiotic recombination intermediates in Saccharomyces cerevisiae. Methods Mol Biol 557: 209-234. https://doi.org/10.1007/978-1-59745-527-5_14

Osman F, Dixon J, Doe CL, Whitby MC (2003) Generating crossovers by resolution of nicked Holliday junctions: a role for Mus81-Eme1 in meiosis. Mol Cell 12:761-774. https://doi.org/10.1016/S10972765(03)00343-5

Park J-M, Intine RV, Maraia RJ (2007) Mouse and human La proteins differ in kinase substrate activity and activation mechanism for tRNA processing. Gene Expr 14:71-81

Petronczki M, Siomos MF, Nasmyth K (2003) Un ménage à quatre: the molecular biology of chromosome segregation in meiosis. Cell 112: 423-440

Petukhova GV, Pezza RJ, Vanevski F, Ploquin M, Masson JY, CameriniOtero RD (2005) The Hop2 and Mnd1 proteins act in concert with Rad51 and Dmc1 in meiotic recombination. Nat Struct Mol Biol 12: 449-453. https://doi.org/10.1038/nsmb923

Phadnis N, Hyppa RW, Smith GR (2011) New and old ways to control meiotic recombination. Trends Genet 27:411-421. https://doi.org/ 10.1016/j.tig.2011.06.007

Rabitsch KP, Gregan J, Schleiffer A, Javerzat J-P, Eisenhaber F, Nasmyth K (2004) Two fission yeast homologs of Drosophila Mei-S332 are required for chromosome segregation during meiosis I and II. Curr Biol 14:287-301. https://doi.org/10.1016/j.cub.2004.01.051

Raffoux X, Bourge M, Dumas F, Martin OC, Falque M (2018) Highthroughput measurement of recombination rates and genetic interference in Saccharomyces cerevisiae. Yeast 35:431-442. https://doi. org/10.1002/yea.3315

Rogers DW, McConnell E, Ono J, Greig D (2018) Spore-autonomous fluorescent protein expression identifies meiotic chromosome missegregation as the principal cause of hybrid sterility in yeast. PLoS Biol 16:e2005066. https://doi.org/10.1371/journal.pbio.2005066

Sabatinos SA, Forsburg SL (2010) Molecular genetics of Schizosaccharomyces pombe. Methods Enzymol 470:759-795. https://doi.org/10.1016/S0076-6879(10)70032-X

Saito TT, Tougan T, Kasama T, Okuzaki D, Nojima H (2004) Mcp7, a meiosis-specific coiled-coil protein of fission yeast, associates with Meu13 and is required for meiotic recombination. Nucleic Acids Res 32:3325-3339. https://doi.org/10.1093/nar/gkh654

Sambrook JF, Russell DW (2000) Molecular cloning: a laboratory manual, 3rd edn. Cold Spring Harbor Laboratory Press, Cold Spring Harbor

Sasanuma H, Tawaramoto MS, Lao JP, Hosaka H, Sanda E, Suzuki M, Yamashita E, Hunter N, Shinohara M, Nakagawa A, Shinohara A (2013) A new protein complex promoting the assembly of Rad51 filaments. Nat Commun 4:1676. https://doi.org/10.1038/ ncomms 2678

Séguéla-Arnaud M, Choinard S, Larchevêque C, Girard C, Froger N, Crismani W, Mercier R (2017) RMI1 and TOP $3 \alpha$ limit meiotic $\mathrm{CO}$ formation through their C-terminal domains. Nucleic Acids Res 45:1860-1871. https://doi.org/10.1093/nar/gkw1210

Sharif WD, Glick GG, Davidson MK, Wahls WP (2002) Distinct functions of S. pombe Rec12 (Spo11) protein and Rec12-dependent crossover recombination (chiasmata) in meiosis I; and a requirement for Rec12 in meiosis II. Cell Chromosome 1:1

Smith GR (2009) Genetic analysis of meiotic recombination in Schizosaccharomyces pombe. Methods Mol Biol 557:65-76. https://doi.org/10.1007/978-1-59745-527-5_6 
Sofueva S, Osman F, Lorenz A, Steinacher R, Castagnetti S, Ledesma J, Whitby MC (2011) Ultrafine anaphase bridges, broken DNA and illegitimate recombination induced by a replication fork barrier. Nucleic Acids Res 39:6568-6584. https://doi.org/10.1093/nar/ gkr340

Straight AF, Belmont AS, Robinett CC, Murray AW (1996) GFP tagging of budding yeast chromosomes reveals that protein-protein interactions can mediate sister chromatid cohesion. Curr Biol 6:1599-1608

Thacker D, Lam I, Knop M, Keeney S (2011) Exploiting sporeautonomous fluorescent protein expression to quantify meiotic chromosome behaviors in Saccharomyces cerevisiae. Genetics 189:423439. https://doi.org/10.1534/genetics.111.131326

Vignard J, Siwiec T, Chelysheva L, Vrielynck N, Gonord F, Armstrong SJ, Schlögelhofer P, Mercier R (2007) The interplay of RecA-related proteins and the MND1-HOP2 complex during meiosis in Arabidopsis thaliana. PLoS Genet 3:1894-1906. https://doi.org/ 10.1371/journal.pgen.0030176

Vincenten N, Kuhl LM, Lam I, Oke A, Kerr ARW, Hochwagen A, Fung J, Keeney S, Vader G, Marston AL (2015) The kinetochore prevents centromere-proximal crossover recombination during meiosis. Elife 4:1-25. https://doi.org/10.7554/eLife.10850

White MA, Petes TD (1994) Analysis of meiotic recombination events near a recombination hotspot in the yeast Saccharomyces cerevisiae. Curr Genet 26:21-30

Wood V, Gwilliam R, Rajandream M-A, Lyne M, Lyne R, Stewart A, Sgouros J, Peat N, Hayles J, Baker S, Basham D, Bowman S, Brooks K, Brown D, Brown S, Chillingworth T, Churcher C, Collins M, Connor R, Cronin A, Davis P, Feltwell T, Fraser A, Gentles S, Goble A, Hamlin N, Harris D, Hidalgo J, Hodgson G,
Holroyd S, Hornsby T, Howarth S, Huckle EJ, Hunt S, Jagels K, James K, Jones L, Jones M, Leather S, McDonald S, McLean J, Mooney P, Moule S, Mungall K, Murphy L, Niblett D, Odell C, Oliver K, O'Neil S, Pearson D, Quail MA, Rabbinowitsch E, Rutherford K, Rutter S, Saunders D, Seeger K, Sharp S, Skelton J, Simmonds M, Squares R, Squares S, Stevens K, Taylor K, Taylor RG, Tivey A, Walsh S, Warren T, Whitehead S, Woodward J, Volckaert G, Aert R, Robben J, Grymonprez B, Weltjens I, Vanstreels E, Rieger M, Schäfer M, Müller-Auer S, Gabel C, Fuchs M, Düsterhöft A, Fritzc C, Holzer E, Moestl D, Hilbert H, Borzym K, Langer I, Beck A, Lehrach H, Reinhardt R, Pohl TM, Eger P, Zimmermann W, Wedler H, Wambutt R, Purnelle B, Goffeau A, Cadieu E, Dréano S, Gloux S, Lelaure V, Mottier S, Galibert F, Aves SJ, Xiang Z, Hunt C, Moore K, Hurst SM, Lucas M, Rochet M, Gaillardin C, Tallada VA, Garzon A, Thode G, Daga RR, Cruzado L, Jimenez J, Sánchez M, del Rey F, Benito J, Domínguez A, Revuelta JL, Moreno S, Armstrong J, Forsburg SL, Cerutti L, Lowe T, McCombie W, Paulsen I, Potashkin J, Shpakovski GV, Ussery D, Barrell BG, Nurse P (2002) The genome sequence of Schizosaccharomyces pombe. Nature 415:871-880. https://doi.org/10.1038/nature724

Yelina NE, Ziolkowski PA, Miller N, Zhao X, Kelly KA, Muñoz DF, Mann DJ, Copenhaver GP, Henderson IR (2013) High-throughput analysis of meiotic crossover frequency and interference via flow cytometry of fluorescent pollen in Arabidopsis thaliana. Nat Protoc 8:2119-2134. https://doi.org/10.1038/nprot.2013.131

Zierhut C, Berlinger M, Rupp C, Shinohara A, Klein F (2004) Mnd1 is required for meiotic interhomolog repair. Curr Biol 14:752-762. https://doi.org/10.1016/j.cub.2004.04.030 